ORIGINAL ARTICLE

\title{
Genotyping serotonin transporter polymorphisms 5-HTTLPR and rs25531 in European- and African-American subjects from the National Institute of Mental Health's Collaborative Center for Genomic Studies
}

\author{
Z Odgerel $^{1,2,6}$, A Talati ${ }^{1,2,6}$, SP Hamilton ${ }^{3}$, DF Levinson ${ }^{4}$ and MM Weissman ${ }^{1,2,5}$
}

A number of studies have suggested DNA sequence variability in the serotonin transporter gene (SLC6A4) between EuropeanAmerican (EA) and African-American (AA) populations, which could be clinically important, given the central role SLC6A4 has in serotonin transmission. However, these studies have had relatively small samples, used self-reported measures of race, and have only tested the promoter-linked polymorphism 5-HTTLPR. Here we genotype 5-HTTLPR and rs25531, a neighboring functional polymorphism, in $954 \mathrm{AA}$ and 2622EA subjects from a National Institute of Mental Health repository sample. Genotyping was performed using fragment analysis by capillary electrophoresis. AA, as compared with $E A$, groups had lower frequencies of the $S$ allele ( 0.25 vs 0.43 ) and SS genotype (0.06 vs 0.19$)$ at 5 -HTTLPR, and higher rates of the $G$ allele at rs 25531 ( $0.21 \mathrm{vs} 0.075)$. A rare $x L$ variant at 5 -HTTLPR was also more common among AAs $(0.017$ vs 0.008$)$. When the polymorphisms were redefined into a high- and low-transcription haplotypes, the AA group showed significantly fewer low-transcription variants $\left(\chi^{2}=4.8, P=0.03\right)$. No genotypes were associated with major depression, any anxiety disorder, or neuroticism in either EA or AA populations. This is the largest study to show SLC6A4 genotype differences between EA and AA populations, and the first to include rs25531. Lack of associations with clinical outcomes may reflect untested moderating environmental influences.

Translational Psychiatry (2013) 3, e307; doi:10.1038/tp.2013.80; published online 24 September 2013

Keywords: African-American; 5-HTTLPR; major depression; rs25531; serotonin transporter

\section{INTRODUCTION}

One of the most widely studied genetic variants in psychiatric research is the repeat-length polymorphism within the promoter region (5-HTTLPR, rs4795541) of the serotonin transporter gene (SLC6A4). 5-HTTLPR occurs primarily as either a shorter sequence (a segment of 14 complex repeats that is associated with lower gene transcription) or a longer one (16 repeats, higher transcription), ${ }^{1}$ although other lengths have also been reported. ${ }^{2}$ Lower frequencies of the short allele (S) have been reported in African-American (AA) compared with European-ancestry (EA) subjects. $^{3-6}$ Given that SLC6A4 has an important role in serotonergic signaling, such allelic differences could be clinically important. However, most studies of 5-HTTLPR in AAs to date have been limited by relatively small sample sizes in part due to the difficulty in recruiting AA subjects in psychiatric, and particularly genetic, research. ${ }^{7}$ Another limitation has been the reliance on self-reported rather than genotypically determined ancestry. Recent evidence also indicates that the transcriptional efficacy of the short and long $(\mathrm{L})$ alleles may be modulated by a singlenucleotide polymorphism, rs25531, that occurs near 5-HTTLPR. ${ }^{8}$ Studies in AAs to date, however, have focused on 5-HTTLPR alone.
This study further characterizes racial differences in the 5-HTTLPR and rs 25531 polymorphisms by taking advantage of the availability of a relatively large and well-characterized sample of AA subjects who were recruited as part of the National Institute of Mental Health's (NIMH's) Molecular Genetics of Schizophrenia (MGS) study. ${ }^{9}$ This was originally a control sample for a schizophrenia study, but additional phenotyping has made it useful for studies of other disorders as well. We genotyped the two SLC6A4 polymorphisms in $954 \mathrm{AA}$ and 2622 EA subjects (after all quality control procedures) for whom clinical data were also available, making this, to our knowledge, the largest genetic sample of AA subjects in which these polymorphisms have been studied. Ancestry was inferred using markers from genome-wide association analyses.

Several large epidemiological studies have reported that rates of major depression are lower in $A A$, as compared with EA populations. ${ }^{10}$ Although there are likely multiple contributing factors, it has not been determined whether one of these factors is variation in serotonin-related polymorphisms. We thus examined whether genotype differences detected in this study might account for clinical differences in rates of depression or anxiety across the racial groups. Our analysis is exploratory, as data were available only for clinical outcomes and not for environmental risk factors, which

\footnotetext{
${ }^{1}$ Department of Psychiatry, Columbia University College of Physicians and Surgeons, New York, NY, USA; ${ }^{2}$ Division of Epidemiology, New York State Psychiatric Institute, New York, NY, USA; ${ }^{3}$ Department of Psychiatry, University of California, San Francisco, CA, USA; ${ }^{4}$ Department of Psychiatry and Behavioral Sciences, Stanford University, Palo Alto, CA, USA and ${ }^{5}$ Department of Epidemiology, Columbia University Mailman School of Public Health, New York, NY, USA. Correspondence: Dr MM Weissman, Division of Epidemiology, Columbia University College of Physicians and Surgeons, Department of Psychiatry, New York State Psychiatric Institute, 1051 Riverside Drive, Unit 24, New York, NY 10032, USA. E-mail: mmw3@columbia.edu

${ }^{6}$ These authors contributed equally to the preparation of this manuscript, and share first-authorship.

Received 17 March 2013; revised 12 June 2013; accepted 15 July 2013
} 
may moderate effects of genetic risks and are sometimes necessary for the development of the clinical phenotype of interest. ${ }^{11,12}$

Although the MGS sample has been widely used in genetics, including GWAS studies, ${ }^{13-15}$ this is the first study to our knowledge to directly genotype 5-HTTLPR in either EA or AA populations of the sample. All genotypes and results from this study will be made available for use by qualified investigators through the NIMH Center for Genetic studies (https://www. nimhgenetics.org).

\section{MATERIALS AND METHODS}

Study population

All subjects were part of the MGS sample of the NIMH Center for Collaborative Genomic Studies on Mental Disorders (https://www.nimhgenetics.org/). DNA was stored at, and provided by, the Rutgers University Cell and DNA repository (RUCDR) (www.rucdr.org; rucdr.rutgers.edu). The sample has been detailed previously. ${ }^{9,16}$

\section{Assessment}

As detailed elsewhere, ${ }^{9}$ all EA and $41 \%$ of AA subjects were recruited by Knowledge Networks, Inc. (Menlo Park, CA, USA) (KN) from a survey research panel that had been recruited using nationwide sampling (random digit dialing), whereas $59 \%$ of AA subjects were recruited by a subcontracting company (Survey Sampling International, Shelton, CT, USA) using banner ads on websites. Subjects were assessed using an on-line self-report version of the Composite International Diagnostic InterviewShort Form (CIDI-SF). Derived from the longer Composite International Diagnostic Interview (CIDI), the CIDI-SF is a structured set of scales assessing eight syndromes: major depressive episodes, panic attacks, agoraphobia, social phobia, specific phobia, generalized anxiety disorder, obsessive compulsive disorder, and alcohol and drug dependence. ${ }^{17}$ We examined major depression and any anxiety disorder (which included generalized anxiety disorder, obsessive compulsive disorder, panic attacks, social anxiety disorder and specific phobia). Questions were assessed on a lifetime basis, with subjects asked to refer to periods of peak symptoms. Initial gate questions were used to skip out subjects least likely to be cases; subjects who were not skipped out then completed remaining questions, and the total number of endorsed symptoms was then dichotomized into categorical diagnoses based on the CIDI-SF algorithms for caseness. ${ }^{18}$ Total classification accuracy, that is, the number of respondents with concordant results on the CIDI and CIDI-SF was 93\% for major depression, and above $96 \%$ for all anxiety disorders. ${ }^{17}$ The parent CIDI has high inter-rater and test-retest reliability ${ }^{19}$ as well as good concordance with other interviewerdetermined DSM diagnoses. ${ }^{20}$ Subjects also completed the neuroticism and extraversion scales of the 12-item short scale of the revised version of the Eysenck Personality Questionnaire (EPQ-R) ${ }^{21}$ Here we examined three outcomes: presence/absence of major depression or of any anxiety disorder and neuroticism score.

Sample

The sample included $2622 \mathrm{EA}$ and 958 AA subjects who had passed all GWAS quality control measures as detailed previously. ${ }^{13}$ DNA was not available for an additional eligible 31 EA and 15 AA subjects. Only 954 AA subjects were included in final analyses, after excluding four with very uncommon 5-HTTLPR variants that were identified here.

AA subjects were on average younger than EA subjects ( 45.3 versus 50.2 years, $t=-9.9, P<0.0001)$ and included a greater proportion of females (61\% vs $\left.52 \%, \chi^{2}=25.1, P<0.0001\right)$. AA subjects were also more likely to have some post-high school education ( $81 \%$ vs $64 \%, \chi^{2}=103.1$, $P<0.0001)$ and to have never been married $\left(28 \%\right.$ vs $15 \%, \chi^{2}, 42.7$, $P<0.0001)$, differences that were likely because of the inclusion in the $A A$ sample of individuals recruited through internet banner ads rather than using survey methods.

\section{Classification of race}

Ancestry of each AA subject was validated using the first principal component score (PC1) that was computed from genome-wide association study data and that reflected a geographical ancestry gradient from north (Europe) to south (Africa). ${ }^{13}$

\section{Genotyping}

DNA was extracted at RUCDR from Epstein-Barr virus-transformed lymphoblastoid cell lines or from fresh blood samples as described elsewhere. ${ }^{15}$ The 5 -HTTLPR and rs25531 polymorphisms of the SLC6A4 gene were genotyped in the two sets of samples. The promoter region of the SLC6A4 gene was amplified in the EA sample using a $10 \mu \mathrm{l}$ of PCR reagent mixture with a forward primer set of $0.25 \mu \mathrm{M}$ VIC-labeled and $0.25 \mu \mathrm{m}$ unlabeled, and $0.50 \mu \mathrm{m}$ reverse primer and $250 \mu \mathrm{m}$ dNTP on the GeneAmp PCR System 9700 (Applied Biosystems, Foster City, CA, USA). PCR primers sequence and reaction conditions have been previously described. ${ }^{22}$ Twenty nanograms of DNA was used as a template. Resulting fragment was resolved in a $2.3 \%$ UltraPure Agarose (Invitrogen, Carlsbad, CA, USA) and visualized under the UV transilluminator. Here, $526 \mathrm{bp}$ and $478 \mathrm{bp}$ fragments were called as a $\mathrm{L}$ and a $\mathrm{S}$ allele at 5 -HTTLPR, respectively. For the A/G SNP rs25531, the PCR amplicon was digested with restriction endonuclease Mspl (New England Biolabs Inc., Boston, MA, USA). The resulting product was analyzed using an ABI3100 DNA Analyzer and the Peak Scan Software v1.0 (Applied Biosystems). Digested fragments with a size of 164-165 bp were determined as a $G$ allele and indigested as an A allele. The AA sample was genotyped using a modified protocol. PCR amplification was performed with $0.50 \mu \mathrm{M}$ VIC-labeled forward primer and $0.50 \mu \mathrm{m}$ reverse primer and was digested with restriction endonuclease Mspl (New England Biolabs Inc.). The resulting product was mixed with the same amount of the PCR amplicon and analyzed using an ABI3100 DNA Analyzer (Applied Biosystems) and Peak Scan Software v1.0

Table 1. Genotype and allele frequencies of the $5-H T T L P R$ and $r s 25531$ polymorphisms in EA and AA populations

\begin{tabular}{|c|c|c|c|c|c|c|c|c|c|}
\hline Populations & & Genc & ype frequenc, & & & $\begin{array}{c}\text { Test of } \\
\text { differences } \\
\text { between all } \\
\text { genotypes }\end{array}$ & $\begin{array}{l}\text { Risk allele } \\
\text { frequency }\end{array}$ & $\begin{array}{c}\text { Rare } \\
\text { allele } \\
\text { frequency }\end{array}$ & $\begin{array}{c}\text { Test of } \\
\text { differences } \\
\text { between all } \\
\text { alleles }\end{array}$ \\
\hline 5-HTTLPR & 'L/L' & 'L/S' & 'L/XL' & 'S/S' & $' x L / S^{\prime}$ & & 'S' & 'xL' & \\
\hline EA & 868 (33.1\%) & $1240(47.39 \%)$ & $1(0.04 \%)$ & $510(19.45 \%)$ & $3(0.11 \%)$ & $\begin{array}{c}\chi^{2}=236.777 \\
\mathrm{df}=4 \\
P=0.0001\end{array}$ & 0.43 & 0.0008 & $\begin{array}{c}\chi^{2}=124.06 \\
\mathrm{df}=2, \\
P=0.0001\end{array}$ \\
\hline rs 25531 & 'A/A' & 'A/G' & ‘G/G' & & & & ‘G' & & \\
\hline EA & 2236 (85.4\%) & 371 (14.2\%) & $12(0.5 \%)$ & & & $\begin{array}{c}\chi^{2}=251.14 \\
\mathrm{df}=2 \\
P=0.0001\end{array}$ & 0.075 & & $\begin{array}{c}\chi^{2}=126.65 \\
\mathrm{df}=1 \\
P=0.0001\end{array}$ \\
\hline AA & $590(61.8 \%)$ & 329 (34.5\%) & 35 (3.7\%) & & & & 0.21 & & \\
\hline
\end{tabular}


(Applied Biosystems). The $L$ and $S$ alleles of 5 -HTTLPR were called by complete-length PCR product and rs 25531 by the Mspl digested product. In order to confirm the result, $10 \%$ of the second set of the samples was resolved in a 2.3\% UltraPure Agarose (Invitrogen, Carlsbad, CA, USA) and visualized under the UV transilluminator. The calling by two different protocols showed $100 \%$ consistent results. The alleles are designated as ' $S$ ' for 14 repeat, 'L' for 16 repeats and 'xL' for 20 repeats. Genotypes will be provided to the NIMH repository and will be made available for use by other qualified investigators.

\section{Statistical analysis}

Hardy-Weinberg equilibrium for the polymorphisms was estimated using the PowerMarker software. ${ }^{23}$ We evaluated the allele, genotype and haplotype distributions between the different ancestry groups and clinical outcomes by means of $\chi^{2}$ statistics using the SAS 9.2 version (SAS Institute Inc, Cary, NC, USA). Associations between genotype and clinical outcomes were evaluated using a logistic regression model, for diagnoses, and linear regressions for neuroticism. All models were adjusted for age and gender. Furthermore, associations between clinical outcomes, genotypes and admixture proportion ( $\mathrm{PC} 1$ ) in the AA population were analyzed using a logistic regression model. Neuroticism scores were analyzed as a continuous variable using a linear regression model. Haplotypic frequencies were estimated using the EM algorithm as implemented PowerMarker software. ${ }^{23}$ Analyses were adjusted for age and sex.

\section{RESULTS}

Genotypes at 5-HTTLPR and rs25531

Genotype and allele frequencies in the full samples did not differ significantly from those predicted by Hardy-Weinberg equilibrium. The genotypic and allelic distributions for 5-HTTLPR and rs25531 in both EA and AA populations are shown in Table 1 and demonstrate significant differences across populations. Specifically, the frequency of the $S$ allele at 5 -HTTLPR was $25 \%$ in $A A$ vs $43 \%$ in EA subjects, and frequency of the $G$ allele at rs 25531 was $7.5 \%$ in AA vs $21 \%$ in EA subjects. The less common $\mathrm{xL}$ allele frequency was $1.65 \%$ in $\mathrm{AA}$ vs $0.08 \%$ in $\mathrm{EA}$. These frequency differences were all statistically significant. In $A A$ subjects, 15-repeat, 18-repeat and 19-repeat alleles at 5-HTTLPR were identified in 1, 1 and 2 subjects, respectively, who were excluded from further analyses.

\section{Haplotype analysis}

Haplotype analyses for 5-HTTLPR and rs25531were performed. EA and $A A$ subjects were significantly different in their frequencies of L-G and S-A, but not L-A, haplotypes (Table 2). As the L-G haplotype is reported to reduce transcription of the serotonin transporter gene similarly to the effect of the $S$ allele, we collapsed all haplotypes into two groups, putative high-transcription ( $L-A$ and $x \mathrm{~L}-\mathrm{A}$ ) vs low-transcription (L-G, S-G, S-A, xL-G) haplotypes, and estimated their frequencies in the two populations. There was a modest but significant difference in the frequencies of high- vs low-transcription haplotypes in AA vs EA subjects (Table 2).

Association of 5-HTTLPR and rs 25531 with psychiatric outcomes Finally, we examined whether 5-HTTLPR and rs25531 alleles and haplotypes were associated with presence/absence of major depression or of anxiety disorders, or with differences in clinical outcomes (specifically, major depression, anxiety disorders, and neuroticism scores), in EA or AA subjects separately, and, if so, whether the genetic differences predicted the rates of these disorders in the populations.

In the AA sample (Table 3a), 286 subjects met lifetime criteria for major depression and 485 for any anxiety disorder. Clinical outcome was not associated with any genotype or haplotype. PC1 scores of AA subjects were not associated with clinical outcomes or with S or G alleles. In EAs, 641 and 1069 met lifetime criteria for major depression and anxiety disorder, respectively (Table $3 \mathrm{~b}$ ). No significant association was found between clinical outcomes and either genotypes or haplotypes.

\section{DISCUSSION}

We report the largest published study of an AA sample to date examining functional 5-HTTLPR and rs25531 polymorphisms within the promoter region of the serotonin transporter gene. Our study shows that, compared with EA subjects, AA subjects have significantly lower rates of the $S$ allele $(0.25$ vs 0.43$)$ and its homozygous genotype SS (0.06 vs 0.19$)$ at 5 -HTTLPR. These rates are consistent with earlier studies. Lotrich et al., ${ }^{5}$ reported that, in a population of 696 self-identified AA subjects, rates of the $S$ allele in the two subsamples were $0.28-0.31(4.1-4.8 \%$ for the SS genotype). In the STAR ${ }^{*} D$ sample (total AA $\left.N=251\right),{ }^{4}$ lower $S$ allele frequencies were observed in AA subjects ( $22 \%$ in antidepressant responders and $20 \%$ in non-responders vs 40 and $42 \%$ in $E A$ subjects), and G allele frequencies (0.26 in AA, 0.08 in EA subjects) were similar to those observed here $(0.21$ and 0.075$)$. Gelernter et $a l^{3}$ found the $S$ allele frequency to be 0.25 in 54 AA subjects vs 0.40 in an EA sample.

We did not find any significant associations between these two serotonin transporter polymorphisms and major depression, anxiety disorders or neuroticism scores in either population alone, nor was there any race-by-genotype-by-diagnosis interaction. There have been inconsistent reports of an association between the $\mathrm{S}$ allele and lifetime risk of major depression, with the largest meta-analysis to date (with $\sim 7800$ cases and 16000 controls from 46 studies) showing a modest effect (odds ratio $=1.076, P=0.001$ ) consistent with a recessive model, but with many indications of possible methodological problems that might account for the results. ${ }^{1}$ Another hypothesis is that the $S$ allele increases risk of depression only through an interaction with life stress. ${ }^{11}$ One recent meta-analysis failed to confirm this effect, ${ }^{24}$ whereas a

\begin{tabular}{|c|c|c|c|c|c|c|}
\hline \multirow[b]{2}{*}{ 5-HTTLPR/rs25531 haplotype } & \multirow[b]{2}{*}{$E A$} & \multirow[b]{2}{*}{$A A$} & \multirow[b]{2}{*}{ Test of difference } & \multicolumn{2}{|c|}{$\begin{array}{c}\text { Haplotype groups by high/low } \\
\text { transcription }^{\mathrm{a}}\end{array}$} & \multirow[b]{2}{*}{ Test of difference } \\
\hline & & & & $E A$ & $A A$ & \\
\hline L-A & 0.50 & 0.52 & $\chi^{2}=115.32, \mathrm{df}=5, P=0.0001$ & 0.50 & 0.53 & $\chi^{2}=4.7838, \mathrm{df}=1, P=0.03$ \\
\hline$x L-A$ & 0.0006 & 0.01 & & & & \\
\hline L-G & 0.07 & 0.21 & & & & \\
\hline S-A & 0.43 & 0.25 & & & & \\
\hline S-G & 0.005 & 0.001 & & & & \\
\hline xL-G & 0.0004 & 0.002 & & 0.50 & 0.46 & \\
\hline
\end{tabular}

Abbreviations: AA, African American; EA, European American; L, long allele; $S$, short allele.

${ }^{a}$ Not bold rows indicate a high-transcription group and bold rows display a low-transcription group. 


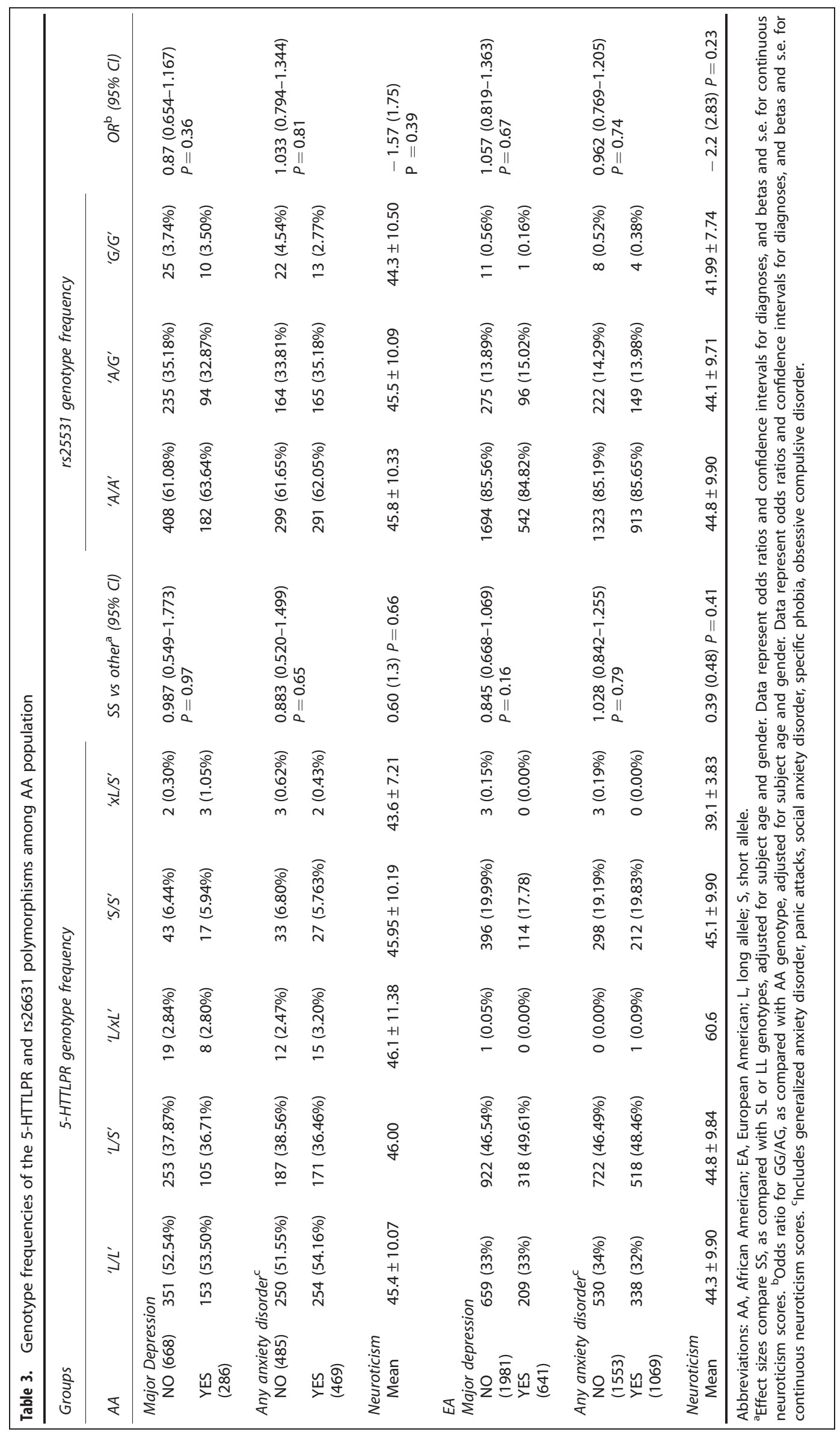


econd one supported $\mathrm{it}^{25}$ with the difference in outcome hinging on whether more restrictive or more inclusive criteria were used to define the phenotype (that is, the type of life stress and its measurement). No environmental measures were available for the present study. There are also several limitations of the present study that are related to the clinical sample. Because subjects were recruited from the community, cases may represent the milder end of the spectrum of depressive and anxiety disorders, for which genetic factors may have a less prominent role. Stronger genetic associations may potentially have been obtained had subjects been recruited from a treatment sample. The on-line diagnostic questionnaire did not provide information about several factors that are thought to predict a greater role for genetic factors, such as family history of depression and details of functional impairment, ${ }^{26,27}$ or conversely a less prominent role, such as the occurrence of depression only in the context of medical disorders, or substance use.

It is also unclear to what extent serotonergic dysregulation leads to depressive phenotypes. Early experiments showed reduced SLC6A4 expression associated with the $S$ variant of 5-HTTLPR when compared with the $L$ variant, but a lack of information on distant regulatory elements could lead to misleading results. ${ }^{28,29}$ Lymphoblastoid cells have an altered gene expression associated with immortalization, and clonal expression of cells in these cultures could result in contradictory findings. ${ }^{30}$ The functional consequences of the genotype differences in multiple cell types remain unclear, and, indeed in postmortem brain, allelic ratios of serotonin transporter mRNA were not correlated with 5-HTTLPR genotype, ${ }^{31,32}$ and PET studies have similarly failed to show serotonin transporter expression in the brain to correlate with allelic status of 5-HTTLPR. ${ }^{3,34}$ It is likely that the presence of the $S$ allele alone is insufficient to cause a clinical phenotype.

The SNP rs25531 is located within a repeat near the polymorphic segment of the 5-HTTLPR, and the G allele reportedly results in reduced transcription levels similar to those of the short allele. $^{8}$ We therefore analyzed phenotypes in relation to lowtranscription ( $S$ and $L-G$ ) and high-transcription (L-A) alleles, but found no significant associations. Martin et al. ${ }^{35}$ reported that there are additional SNPs that contribute to transcriptional variation of the serotonin transporter gene, but these are relatively rare so that larger samples would be required to detect any phenotypic effects. It is of interest that when the $\mathrm{L}$ allele containing the $\mathrm{G}$ allele of $\mathrm{rs} 25531$ was assumed to have low transcription, the total frequency of low-transcription ( $S$ and L-G) alleles was similar in AA (46\%) and EA (50\%) subjects, despite the substantial difference in $S$ allele frequencies. All polymorphisms were in Hardy-Weinberg equilibrium. Assuming that these polymorphisms do have effects on expression in an at least some tissues, one speculative interpretation of this finding would be as follows. Both historical populations have experienced similar adaptive selective pressures that have shaped SLC6A4 expression levels. The result has been similar overall frequencies of high- and low-transcription common polymorphisms, but with the $\mathrm{S}$ allele (more common in Europeans) apparently partially replacing L-G (more common in Africans) over time. One might assume that alleles with large deleterious effects (such as a major increase in risk of severe depression) would have been subjected to negative selection, whereas the observed common polymorphisms may be relevant to the normal regulation of SLC6A4 expression.

\section{CONFLICT OF INTEREST}

In the past two years, Dr Weissman received funding from the NIMH, NIDA, NARSAD the Sackler Foundation, the Templeton Foundation and the Interstitial Cystitis Association, and received royalties from the Oxford University Press, Perseus Press, the American Psychiatric Association Press and MultiHealth Systems. None of these present a conflict of interest with this manuscript. The remaining authors declare no conflict of interest.

\section{ACKNOWLEDGEMENTS}

Access to the sample was provided as part of an NIMH program project P01MH60970 (Weissman, PI). The present study is supported by NIMH Conte Center 1P50MH090966 (Jay Gingrich, MD, PhD, PI) and 1RC2MH089916 (M Weissman, PI). Dr Talati is funded by K01DA029598. Biomaterials and phenotypic data were obtained from the following projects that participated in the NIMH Control Samples: Control subjects from the National Institute of Mental Health Schizophrenia Genetics Initiative (NIMH-Gl), data and biomaterials are being collected by the 'Molecular Genetics of Schizophrenia II' (MGS-2) collaboration. The investigators and coinvestigators are ENH/Northwestern University, Evanston, IL, MH059571, Pablo V. Gejman, MD (Collaboration Coordinator; PI), Alan R. Sanders, MD; Emory University School of Medicine, Atlanta, GA,MH59587, Farooq Amin, MD (PI); Columbia University, M Jay A Gingrich, MD (PI); Louisiana State University Health Sciences Center; New Orleans, Louisiana, MH067257, Nancy Bueaola, APRN, BC, MSN. (PI); University of California-Irvine, Irvine, CA, MH60870, William Byerley, MD (PI); Washington University, St Louis, MO, U01, MH060879, C Robert Cloninger, MD (PI); University of lowa, lowa, IA,MH59566, Raymond Crowe, MD (PI), Donald Black, MD; University of Colorado, Denver, CO, MH059565, Robert Freedman, MD (PI); Stanford University, Stanford, CA, MH061675 and MH089916, Douglas Levinson MD (PI); University of Queensland, Queensland, Australia, MH059588, Bryan Mowry, MD (PI); Mt. Sinai School of Medicine, New York, NY,MH59586, Jeremy Silverman, PhD (PI). The samples were collected by Vishwajit Nimgaonkar's group at the University of Pittsburgh, as part of a multi-institutional collaborative research project with Jordan Smoller, MD, DSc., and Pamela Sklar, MD, PhD, Massachusetts General Hospital (grant MH 63420). Data and biomaterials used in Study 23 were collected by the University of Pittsburgh and funded by an NIMH grant (Genetic Susceptibility in Schizophrenia, MH 56242) to Vishwajit Nimgaonkar, MD, PhD Additional Principal Investigators on this grant include Smita Deshpande, MD, Dr Ram Moanohar Lohia Hospital, New Delhi, India; and Michael Owen, MD, PhD, University of Wales College of Medicine, Cardiff, UK.

\section{REFERENCES}

1 Clarke H, Flint J, Attwood AS, Munafo MR. Association of the 5- HTTLPR genotype and unipolar depression: a meta-analysis. Psychol Med 2010; 40: 1767-1778.

2 Nakamura M, Ueno S, Sano A, Tanabe H. The human serotonin transporter gene linked polymorphism (5-HTTLPR) shows ten novel allelic variants. Mol Psychiatry 2000; 5: 32-38.

3 Gelernter J, Kranzler H, Coccaro EF, Siever LJ, New AS. Serotonin transporter protein gene polymorphism and personality measures in African American and European American subjects. Am J Psychiatry 1998; 155: 1332-1338.

4 Kraft JB, Peters EJ, Slager SL, Jenkins GD, Reinalda MS, McGrath PJ et al. Analysis of association between the serotonin transporter and antidepressant response in a large clinical sample. Biol Psychiatry 2007; 61: 734-742.

5 Lotrich FE, Pollock BG, Ferrell RE. Serotonin transporter promoter polymorphism in African Americans: allele frequencies and implications for treatment. Am J Pharmacogenomics 2003; 3: 145-147.

6 Patkar AA, Berrettini WH, Hoehe M, Thornton CC, Gottheil E, Hill K et al. Serotonin transporter polymorphisms and measures of impulsivity, aggression, and sensation seeking among African-American cocaine-dependent individuals. Psychiatry Res 2002; 110: 103-115.

7 Murphy EJ, Wickramaratne P, Weissman MM. Racial and ethnic differences in willingness to participate in psychiatric genetic research. Psychiatr Genet 2009; 19: 186-194.

8 Hu XZ, Lipsky RH, Zhu G, Akhtar LA, Taubman J, Greenberg BD et al. Serotonin transporter promoter gain-of-function genotypes are linked to obsessivecompulsive disorder. Am J Hum Genet 2006; 78: 815-826.

9 Sanders AR, Levinson DF, Duan J, Dennis JM, Li R, Kendler KS et al. The Internetbased MGS2 control sample: self report of mental illness. Am J Psychiatry 2010; 167: 854-865.

10 Williams DR, Gonzalez HM, Neighbors H, Nesse R, Abelson JM, Sweetman J et al. Prevalence and distribution of major depressive disorder in African Americans, Caribbean blacks, and non-Hispanic whites: results from the National Survey of American Life. Arch Gen Psychiatry 2007; 64: 305-315.

11 Caspi A, Sugden K, Moffitt TE, Taylor A, Craig IW, Harrington H et al. Influence of life stress on depression: moderation by a polymorphism in the $5-\mathrm{HTT}$ gene. Science 2003; 301: 386-389.

12 Munafo MR, Durrant C, Lewis G, Flint J. Gene X environment interactions at the serotonin transporter locus. Biol Psychiatry 2009; 65: 211-219.

13 Shi J, Levinson DF, Duan J, Sanders AR, Zheng Y, Pe'er I et al. Common variants on chromosome 6p22.1 are associated with schizophrenia. Nature 2009; 460: 753-757. 
14 Kendler KS, Kalsi G, Holmans PA, Sanders AR, Aggen SH, Dick DM et al. Genomewide association analysis of symptoms of alcohol dependence in the molecular genetics of schizophrenia (MGS2) control sample. Alcohol Clin Exp Res 2011; 35: 963-975.

15 Levinson DF, Duan J, Oh S, Wang K, Sanders AR, Shi J et al. Copy Number Variants in Schizophrenia: Confirmation of Five Previous Findings and New Evidence for 3q29 Microdeletions and VIPR2 Duplications. AJ Psychiatry 2011; 168 302-316.

16 Talati A, Fyer AJ, Weissman MM. A comparison between screened NIMH and clinically interviewed control samples on neuroticism and extraversion. $\mathrm{Mol}$ Psychiatry 2008; 13: 122-130.

17 Kessler R, Andrews G, Mrozcek D, Ustun B, Wittchen H. The World Health Organization Composite International Diagnostic Interview Short-Form (CIDI-SF). Int J Methods Psychiatr Res 1996; 7: 171-185.

18 Walters E, Kessler RC, Nelson CB, Mroczek DC. Scoring the World Health Organization's Composite International Diagnostic Interview Short Form Form (CIDI-SF) (revised December 2002). World Health Organization: Geneva, 2002.

19 Wittchen HU. Reliability and validity studies of the WHO-Composite International Diagnostic Interview (CIDI): a critical review. J Psychiatr Res 1994; 28: 57-84.

20 Janca A, Robins LN, Bucholz KK, Early TS, Shayka JJ. Comparison of Composite International Diagnostic Interview and clinical DSM-III-R criteria checklist diagnoses. Acta Psychiatr Scand 1992; 85: 440-443.

21 Eysenck S, Eysenck HJ, Barrett P. A revised version of the psychoticism scale. Personal Individual Diff 1985; 6: 21-29.

22 Kraft JB, Slager SL, McGrath PJ, Hamilton SP. Sequence analysis of the serotonin transporter and associations with antidepressant response. Biol Psychiatry 2005 58: $374-381$

23 Liu K, Muse SV. PowerMarker: an integrated analysis environment for genetic marker analysis. Bioinformatics 2005; 21: 2128-2129.

24 Risch N, Herrell R, Lehner T, Liang KY, Eaves L, Hoh J et al. Interaction between the serotonin transporter gene (5-HTTLPR), stressful life events, and risk of depression: a meta-analysis. JAMA 2009; 301: 2462-2471.

25 Karg K, Burmeister M, Shedden K, Sen S. The serotonin transporter promoter variant (5-HTTLPR), stress, and depression meta-analysis revisited: evidence of genetic moderation. Arch Gen Psychiatry 2011; 68: 444-454.
26 Holmans P, Zubenko GS, Crowe RR, DePaulo Jr JR, Scheftner WA, Weissman MM et al. Genomewide significant linkage to recurrent, early-onset major depressive disorder on chromosome 15q. Am J Hum Genet 2004; 74: 1154-1167.

27 Mondimore FM, Zandi PP, Mackinnon DF, Mclnnis MG, Miller EB, Crowe RP et al. Familial aggregation of illness chronicity in recurrent, early-onset major depression pedigrees. Am J Psychiatry 2006; 163: 1554-1560.

28 Heils A, Teufel A, Petri S, Stober G, Riederer P, Bengel D et al. Allelic variation of human serotonin transporter gene expression. J Neurochem 1996; 66: 2621-2624.

29 Lesch KP, Bengel D, Heils A, Sabol SZ, Greenberg BD, Petri S et al. Association of anxiety-related traits with a polymorphism in the serotonin transporter gene regulatory region. Science 1996; 274: 1527-1531.

30 Plagnol V, Uz E, Wallace C, Stevens H, Clayton D, Ozcelik T et al. Extreme clonality in lymphoblastoid cell lines with implications for allele specific expression analyses. PLOS ONE 2008; 3: e2966.

31 Sugden K, Tichopad A, Khan N, Craig IW, D'Souza UM. Genes within the serotonergic system are differentially expressed in human brain. BMC Neurosci 2009; 10: 50.

32 Lim JE, Papp A, Pinsonneault J, Sadée W, Saffen D. Allelic expression of serotonin transporter (SERT) mRNA in human pons: lack of correlation with the polymorphism SERTLPR. Mol Psychiatry 2006; 11: 649-662.

33 Murthy NV, Selvaraj S, Cowen PJ, Bhagwagar Z, Riedel WJ, Peers P et al. Serotonin transporter polymorphisms (SLC6A4 insertion/deletion and rs25531) do not affect the availability of $5-\mathrm{HTT}$ to $[11 \mathrm{C}]$ DASB binding in the living human brain. Neuroimage 2010; 52: 50-54.

34 Parsey RV, Ojha A, Ogden RT, Erlandsson K, Kumar D, Landgrebe M et al. Metabolite considerations in the in vivo quantification of serotonin transporters using 11C-DASB and PET in humans. J NuCl Med 2006; 47: 1796-1802.

35 Martin J, Cleak J, Willis-Owen SA, Flint J, Shifman S. Mapping regulatory variants for the serotonin transporter gene based on allelic expression imbalance. Mol Psychiatry 2007; 5: 421-422.

c) This work is licensed under a Creative Commons AttributionBY NA SA Commercial-ShareAlike 3.0 Unported License. To view a copy of this license, visit http://creativecommons.org/licenses/by-nc-sa/3.0/ 